\title{
Macroscopic Simulation of Deformation in Soft Microporous Composites
}

\author{
Jack D. Evans and François-Xavier Coudert* \\ Chimie ParisTech, PSL Research University, CNRS, Institut de Recherche de Chimie Paris, 75005 Paris, \\ France \\ E-mail: fx.coudert@chimie-paristech.fr
}




\begin{abstract}
Soft microporous materials exhibit properties, such as gated adsorption and breathing, which are highly desirable for many applications. These properties are largely studied for single crystals, however, many potential applications expect to construct structured or composite systems, examples of which include monoliths and mixed-matrix membranes. Herein, we use finite element methods to predict the macroscopic mechanical response of composite microporous materials. This implementation connects the microscopic treatment of crystalline structures to the response of a macroscopic sample. Our simulations reveal the bulk modulus of an embedded adsorbent within a composite is affected by the thickness and properties of the encapsulating layer. Subsequently, we employ this methodology to examine mixed-matrix membranes and materials of negative linear compressibility. This application of finite element methods allows for unprecedented insight into the mechanical properties of real-world systems and supports the development of composites containing mechanically anomalous porous materials.
\end{abstract}

\title{
Graphical TOC Entry
}

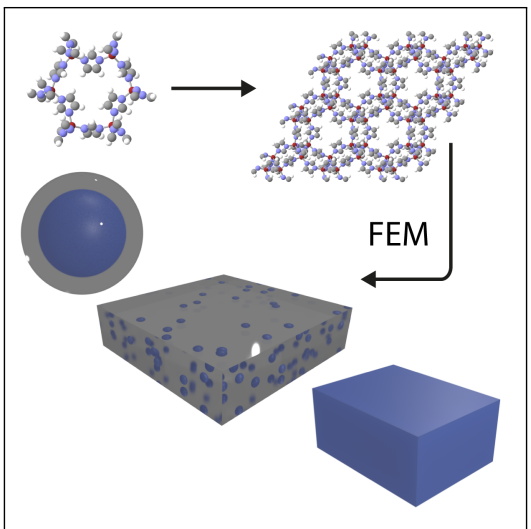


Soft microporous materials have demonstrated excellent potential for use in separation, drug delivery and sensing applications.14 Particularly, stimuli-responsive materials can exhibit curious and counter-intuitive properties which have garnered significant experimental and theoretical investigation. $\frac{5}{5}$ One such phenomenon is that of adsorption-induced deformation, ${ }^{6}$ where the adsorption of guest species exerts stress on the porous system causing a deformation (or strain) of the microporous structure. This phenomenon can result, for the specific cases of very soft or highly asymmetric frameworks, in eye-catching experimental observations such as gated adsorption, breathing and negative gas adsorption. ${ }^{7-9}$

Understanding the response of porous materials under physical or chemical stimulation requires a good understanding of the mechanical behavior of the host material itself. The atomistic mechanisms behind these phenomena can be examined in detail using simulation methods at the microscopic scale. ${ }^{10}$ Notably, high accuracy density functional theory (DFT) methods have proven useful in probing this mechanical behavior of microporous materials, in and outside their elastic regime, by correlating atomistic stresses and strains in the fully periodic (and perfect) crystal structure - as illustrated in Figure 1 These methods have been used to uncover the mechanical properties of many crystalline materials resulting in valuable insight into negative linear compressibility, ${ }^{111}$ auxeticity, ${ }^{[12}$ and chiral induction. ${ }^{13}$ However, these conventional methods are limited to single crystals of pure phase materials. On the other hand, practical applications of porous crystals are often used within composite materials, such as mixed-matrix membranes (MMMs) $)^{14115}$ or thin films anchored on a support.16]17

Herein, we describe the application of finite element methods (FEM) $\frac{18}{18}$ to examine the macroscopic mechanics of crystalline and nanocomposite materials, Figure 1 FEM are powerful numerical techniques for the resolution of boundary value problems for partial differential equations, which subdivide a large complex problem into small parts that are simpler to solve. In this study, we use it to perform structural mechanics analyses, i.e. solving for deformation and stresses in structures such as an entire single crystal and composites. This results in unprecedented insight into the properties of composite materials, beyond the previous approaches focusing on the periodic lattice or the unit cell. In addition to a fundamental advance, this approach is relevant for practical applications whereby encapsulation has been shown to attenuate deformation $\frac{19}{21}$ and thus diminish the often highly-desired flexible response. Importantly, this property could be potentially leveraged to tune the transition pressure of gated adsorption, Figure $1 \mathrm{~b}, \sqrt{22}$ for energyefficient separations. 23

In general, the description of a material's volumetric response to adsorption-induced stress can be simply described, in the linear regime, by Hooke's law:

$$
\epsilon=\frac{\sigma_{\mathrm{A}}}{K_{\mathrm{A}}}
$$



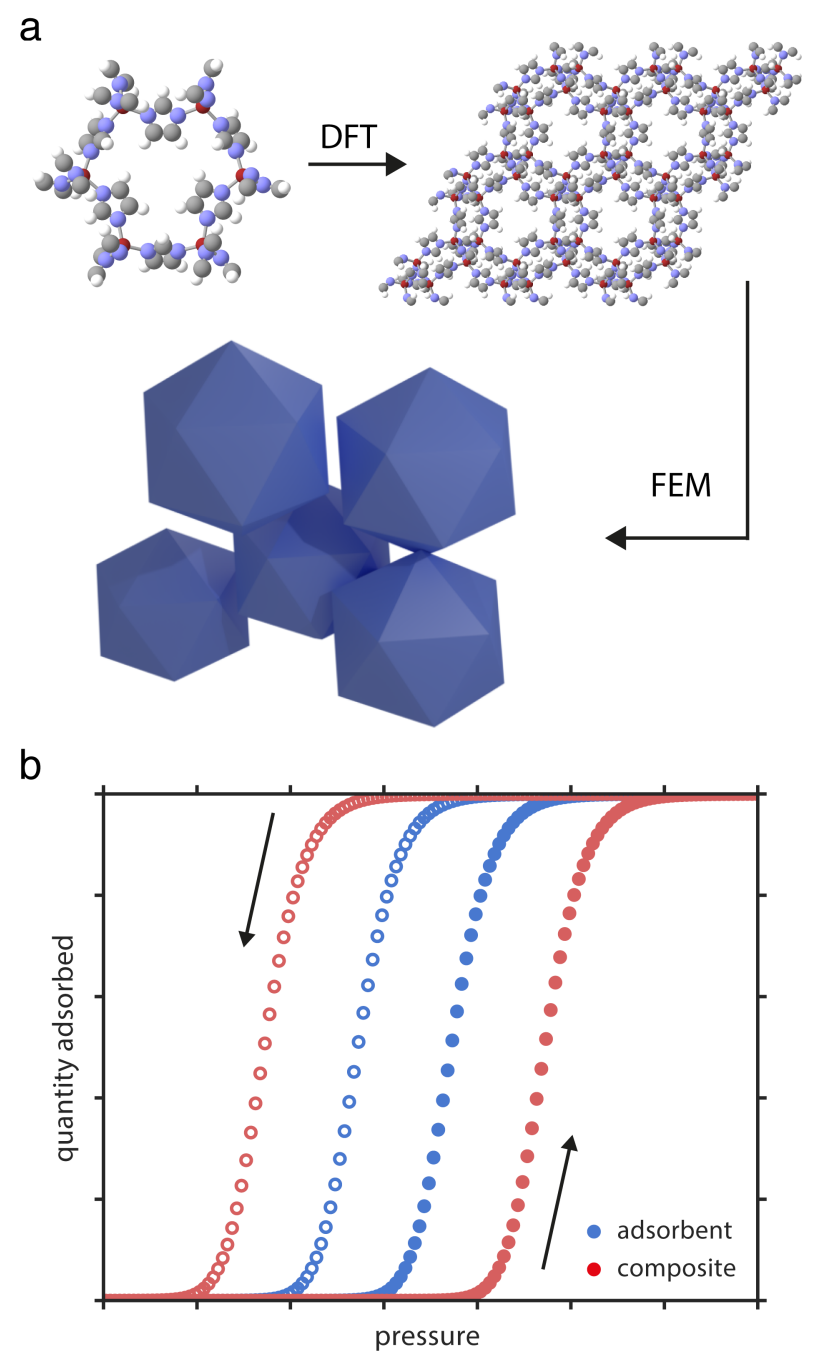

Figure 1: (a) Density functional theory (DFT) methods are employed to relate atomistic strains to a periodic lattice and, in the present work, we have used finite element methods (FEM) to extend this description to the macroscopic scale of an entire crystal. (b) A hypothetical description of gated adsorption in an isolated flexible adsorbent (blue) and an adsorbent surrounded by an elastic matrix (red), adsorption is indicated by filled symbols and desorption empty symbols. 
where $\epsilon$ is volumetric strain, $\sigma_{\mathrm{A}}$ is adsorption stress and $K_{\mathrm{A}}$ is the bulk modulus of the adsorbing material. This relation is accurate for the behavior of flexible crystals in the absence of an encapsulating phase. However, the analytical expression for volumetric strain is more complex for a composite system, and would have to depend on the properties of all phases present, the nature of their interface, as well as the geometry of the composite. Recently, Neimark and coworkers developed analytical models, using well-established laws of elasticity, to quantify how an encapsulating matrix results in an attenuation of the adsorption-induced stress and thus deformation. ${ }^{22}$ This was achieved for a simple spherical model of a single particle, surrounded by an external medium - itself spherical or infinite - to give expressions for three specific cases, which are displayed in Figure 2. Here, we summarize the results obtained by this earlier work, and which will serve as a point of comparison for our numerical investigation.

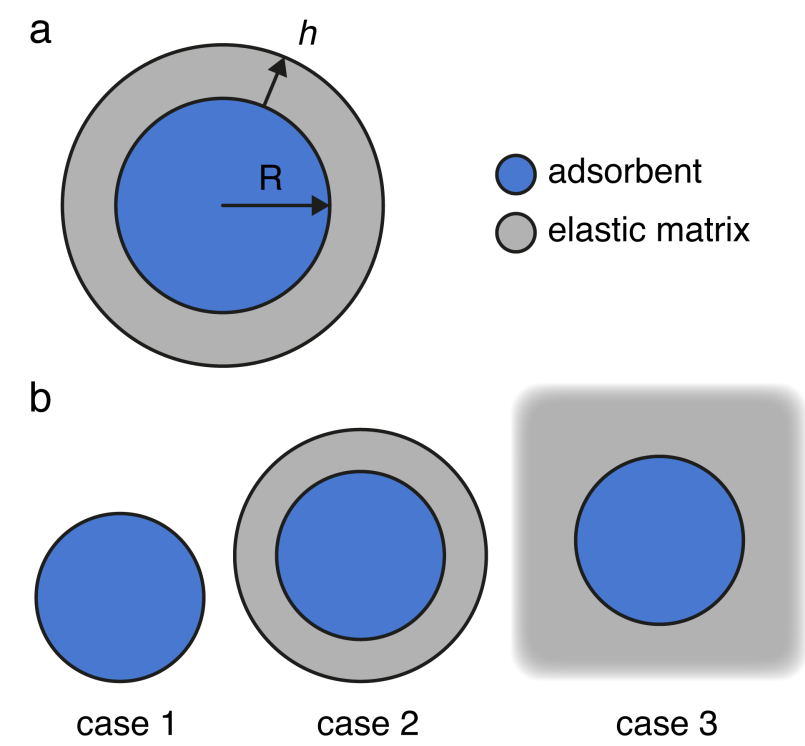

Figure 2: (a) The model system used by Neimark and coworkers to represent a composite material. 22$]$ (b) Three cases for which analytic descriptions have been reported: case 1, an unconfined adsorbent; case 2, an adsorbent surrounded by a finite encapsulating shell; case 3, an adsorbent in an infinite matrix.

The soft porous crystal alone, i.e. an unconfined adsorbent under no external pressure (case 1), is well described by Hooke's law, Equation 1 1 . This is the standard volumetric response of any material to external stress, whether that is an external mechanical stress or a stress exerted "from inside" and due to adsorption. For a spherical adsorbent surrounded by another material (case 2) this standard response is decreased by the confinement, and its expression is given by:

$$
\epsilon=\frac{\sigma_{\mathrm{A}}}{K_{\mathrm{A}}}\left(1-\frac{1}{1+K_{\mathrm{A}}\left(\frac{g}{3 K}+\frac{1-g}{4 G}\right)}\right)
$$


where $K$ and $G$ are the bulk and shear moduli of the surrounding material, and $g=\frac{R^{3}}{(R+h)^{3}}$ characterizes the volumetric ratio of the adsorbent and surrounding matrix.

There exists a special instance of case 2 where the adsorbent is surrounded by a non-deformable outer container. Physically, this represents a composite where the surrounding material is much stiffer. This results in different boundary conditions and a different expression for the expected deformation, which is then given by:

$$
\epsilon=\frac{\sigma_{\mathrm{A}}}{K_{\mathrm{A}}}\left(\frac{1}{1+\frac{g K}{K_{\mathrm{A}}}+\frac{4 G(1+g)}{3 K_{\mathrm{A}}}}\right)
$$

Finally, another case is that of a spherical adsorbent in an infinite elastic matrix (case 3), which is a simple model corresponding to industrially relevant MMMs - it is equivalent to a MMM with a low concentration of soft crystal particles. In this case the volumetric strain was found to be reduced by a constant factor:

$$
\epsilon=\frac{\sigma_{\mathrm{A}}}{K_{\mathrm{A}}}\left(\frac{1}{1+\frac{4 G}{K_{\mathrm{A}}}}\right)
$$

While composite microporous materials have been investigated with respect to adsorption and gas separation, 2425 the above analytical expressions, developed by Neimark and coworkers, give fundamental insight into their expected mechanical properties. These expressions, however, feature some approximations and are limited to spherical isotropic models and there is thus a need for numerical simulation methods to describe the response of anisotropic crystals, more complex systems and realistic nanostructured composites. In this study, we first investigate the mechanical response of similar simple spherical systems using our FEM approach. Subsequently, we apply this method to physically relevant and more complex models of MMMs and a nanocomposite containing a crystal exhibiting negative linear compressibility (NLC).

Firstly, in order to both validate the FEM approach and probe the validity of the analytic expressions derived by Neimark et al., a series of simulations were performed for simple spherical models displayed in Figure $3 \mathrm{k}$. The structural models are comprised of a spherical adsorbent particle with a radius of $0.4 \mu \mathrm{m}$ and a surrounding shell of a height between $0 \mu \mathrm{m}$ (no matrix) and $10 \mu \mathrm{m}$ (much larger than the adsorbent's radius). The adsorbent and surrounding matrix were simulated with the properties defined in Table 1 These parameters were chosen to represent the mechanical properties of frequently studied systems, ${ }^{26 \mid 27}$ in particular, ZIF-8 ${ }^{28}$ and mesoporous silica (adsorbent and matrix 1, respectively). Notably, we do not consider the porosity explicitly by these simulations instead we consider the soft and unique elastic properties which are exhibited by these materials.

Figure $3 \mathrm{p}$ illustrates the volumetric strain observed for an adsorption stress applied to the adsorbent material, in a way similar to a classical mechanical stress-strain curve. For an unconfined adsorbent under 
Table 1: Material properties of the adsorbent and three encapsulating matrix materials considered in this investigation.

\begin{tabular}{lllll}
\hline material & $\rho / \mathrm{kg} \cdot \mathrm{m}^{-3}$ & $K / \mathrm{GPa}$ & $G / \mathrm{GPa}$ & $\nu$ \\
\hline adsorbent & 924.3 & 2.895 & 1.110 & 0.33 \\
matrix 1 & 242.0 & 8.333 & 3.846 & 0.30 \\
matrix 2 & 242.0 & 0.8333 & 0.3846 & 0.30 \\
matrix 3 & 242.0 & 41.67 & 19.23 & 0.30 \\
\hline
\end{tabular}

a

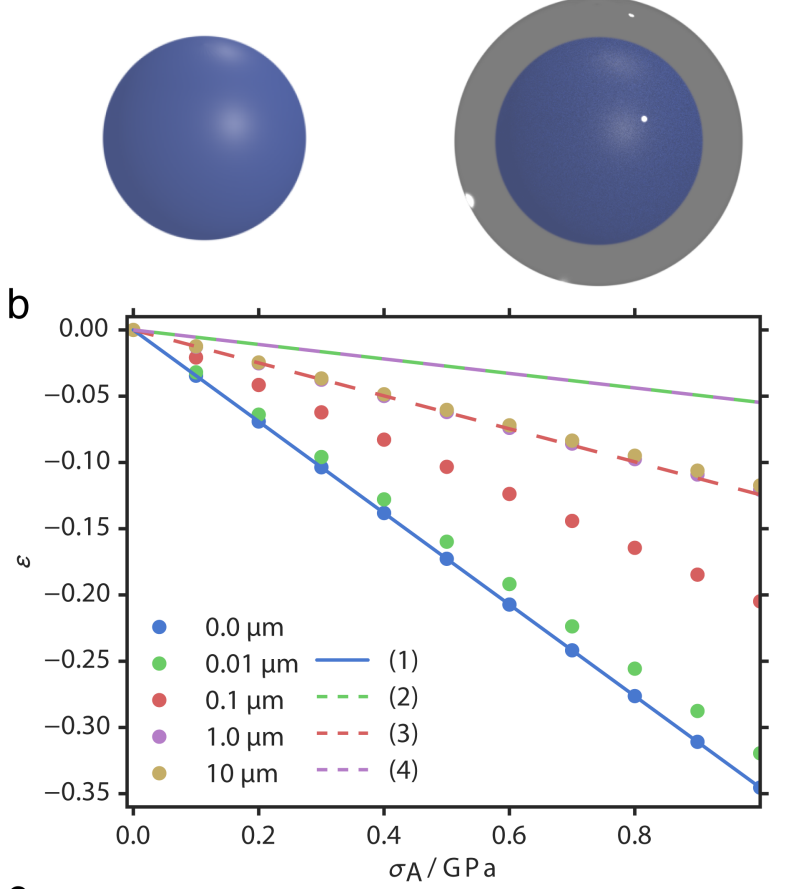

C

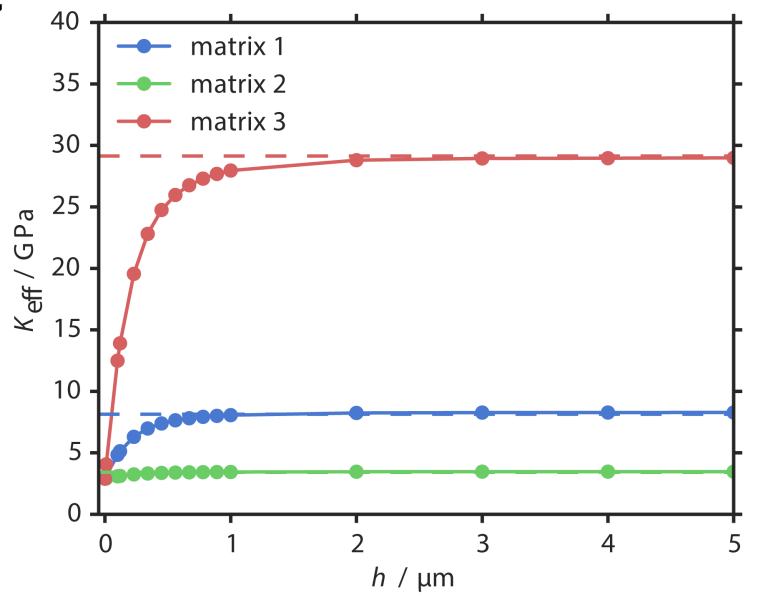

Figure 3: (a) The simple three-dimensional spherical models investigated: unconfined adsorbent particle (left) and composite system (right). (b) Volumetric strain for an adsorbent with an encapsulating shell of matrix 1 (points) where the width, $h$, corresponds to the color code and the prediction of the analytical models are compared (lines). (c) Effective bulk modulus of the adsorbent $\left(K_{\text {eff }}\right)$ for composites comprised of the adsorbent with radius $0.4 \mu \mathrm{m}$ and a shell of width $h$ for matrix 1 , matrix 2 , or matrix 3 (blue, green, red; respectively). 
adsorption stress, we observe the expected response corresponding to Hooke's law, Equation 1. The composite models, however, show reduced strain owing to the encapsulating sphere constructed of stiffer material (matrix 1) with a bulk modulus $8.33 \mathrm{GPa}$, greater than that of the adsorbent (3.3 GPa). The extent of reduction is observable for a shell of thickness $0.01 \mu \mathrm{m}$, i.e. $2 \%$ of the radius of adsorbent. The magnitude of this effect reaches a maximum for a width of $1.0 \mu \mathrm{m}$, with larger widths showing no greater reduction in strain. Strains computed by FEM simulation were compared to that expected from the analytical models reported previously. We compare the strain elucidated for the maximum attenuation achieved, for a width of $10 \mu \mathrm{m}$, in Figure $3 \mathrm{p}$. The models for a flexible sphere and an infinite matrix, Equation 2 and Equation 4 significantly overstate the reduction observed for the composite material. However, we find a good agreement for Equation 3 corresponding to the case where the external boundary of the matrix is fixed. The source of the discrepancy is likely to result from the simplified boundary conditions chosen during construction of the analytical expressions.

This FEM study has highlighted that the maximum attenuation achieved by the construction of a composite can be described by Equation 3. We find this is accurate for composites comprised of either softer or harder materials, such as matrix 2 and matrix 3 . We can thus define for the nanocomposite system an effective bulk modulus $\left(K_{\text {eff }}\right)$ of the adsorbent from the linear stress-strain behavior. Figure 3 c demonstrates the evolution of $K_{\text {eff }}$ for an increasing width of encapsulating material. Notably, we find that even an extremely soft material, such as matrix 2 - softer than the adsorbent itself - yields an increase in effective bulk modulus of the adsorbent from 2.89 GPa to $3.47 \mathrm{GPa}$. This significant increase would, in practical applications, influence the flexible adsorption properties of the adsorbent and thus its performance. In particular, this demonstrates the advantageous mechanical stability observed for composites containing soft adsorbents. 29

Ultimately, the results from these numerical FEM simulations parallel the result of the analytical model - the addition of an encapsulating material can be used to tune the mechanical response of an adsorbent. We find that Equation 3 shows good agreement to the strain resulting from adsorption stress in a spherical composite material. Furthermore, the numerical simulations reveal shortcomings of the analytical models for other cases. Subsequently, we have applied this FEM approach to provide insight into two complex and experimentally relevant systems.

Nanocomposite membrane systems, such as MMMs, are desirable for application as they combine the processability of polymers with the gas separation properties of crystalline frameworks producing desirable nanostructured membrane materials. Separation methods employing membranes are more energy efficient than conventional separations ${ }^{30}$ and the use of flexible adsorbent materials has the potential to significantly improve the energy efficiency of gas separations. 23

As demonstrated for simple spherical models the formation of a composite has implications for the 
mechanical properties of the adsorbent and unsurprisingly we expect this to be observed in MMMs.21 To investigate this effect, we produced models of MMMs - illustrated in Figure 4 4 - where a number of adsorbent particles of fixed radius $(10 \mu \mathrm{m}$ or $20 \mu \mathrm{m})$ are randomly dispersed throughout the volume of the encapsulating polymer. The material's properties for the adsorbent are identical to that of the simple spherical model, while for the membrane we use the mechanical properties of "matrix 1". Subsequently, the number of adsorbent particles was varied to result in models with increasing volumetric fraction (or loading). Because the systems were generated with a random dispersion of adsorbent particles for each set of parameters 10 unique simulations were completed at each loading to obtain a representative average. The importance of this methodology is demonstrated for membranes of a low volumetric fraction where the small sample size resulted in large variances.

From the simulation of the stress-strain curves in each case, we computed the effective bulk modulus of the adsorbent for a given volumetric fraction of particles in the composite system displayed in Figure $4 \mathrm{~b}$. Adsorbents of radius $10 \mu \mathrm{m}$, despite showing large variance at a low volumetric fraction, exhibit an average effective bulk modulus similar to that expected for a sphere surrounded by a much larger material, described by Equation 3. However, dispersed within membranes with greater volumetric fraction this effect of the surrounding matrix is diminished. Adsorbents at a volumetric fraction of $10.5 \%$ have an effective bulk modulus of $7.04 \mathrm{GPa}$ reduced more than $10 \%$ from $7.95 \mathrm{GPa}$ observed at $0.03 \%$. This trend is reproduced for larger sized adsorbents although the effective bulk modulus in these cases is noticeably reduced.

The simulations presented here demonstrate the effect that dispersing adsorbents within a matrix can have on the mechanical properties. The large variance observed at low volume fractions suggests than the action for this effect is related to the position of adsorbent within the matrix. To investigate this further, simulations of an individual adsorbent positioned at points centered at various positions through the $400 \mathrm{\mu m}$ $\times 400 \mu \mathrm{m}$ face of the membrane model were conducted. Figure 4 depicts the evolution of the effective bulk modulus of the adsorbent as a function of its $z$ coordinate within the membrane. When placed on the surface of the matrix the adsorbent, as expected, shows no change in bulk modulus. Within the surface of the matrix, the effect of the surrounding material is sudden with the adsorbent displaying stiffer mechanical properties even when not completely encapsulated. Furthermore, the amount of surrounding material is observed to have a considerable result on the bulk modulus. Adsorbents positioned barely within the matrix show a softer response than those placed in the center of the membrane structure. This is demonstrated clearly for the larger adsorbent, with a diameter of $40 \%$ the width of the matrix, here the bulk modulus is unable to be strengthened to the same extent as the smaller adsorbent. Ultimately, the edge effects illustrated in Figure 4 . manifest the volume fraction trend observed in the MMM models. At high concentration of particles there are more positioned at the edges of the structure resulting in a decreased effect of encapsulation. 
a
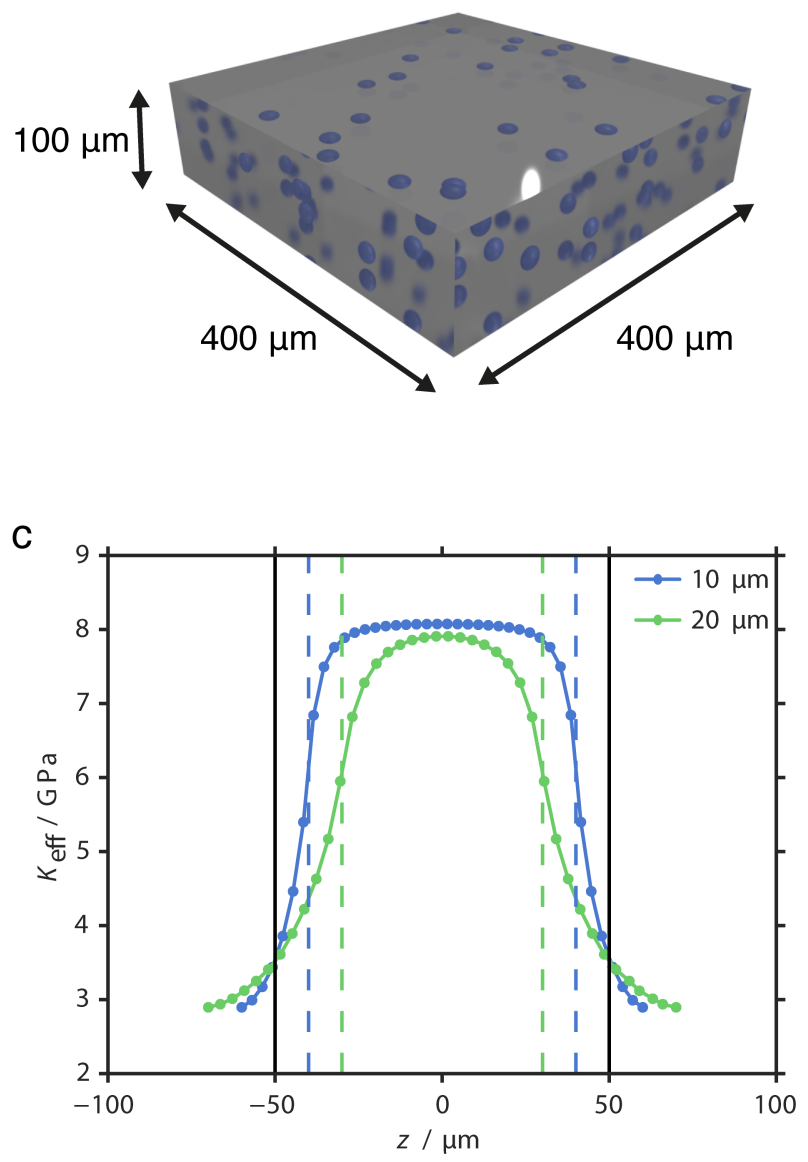

b
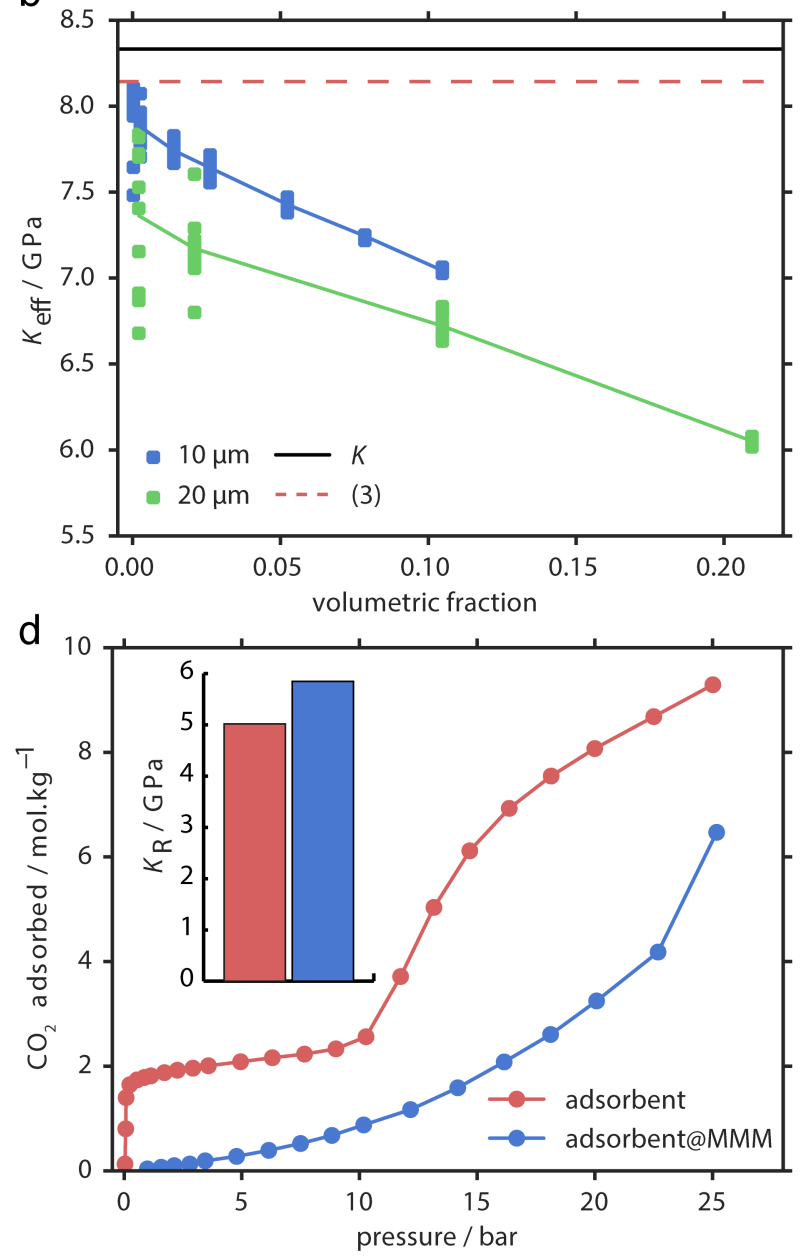

Figure 4: (a) Illustration and dimensions of one realization of the MMM model used in this study. (b) Effective bulk modulus of the adsorbent $\left(K_{\text {eff }}\right)$ for a given volumetric fraction in the membrane. The individual simulations are displayed by points and lines are drawn between the averages for each volumetric fraction. (c) Effective bulk modulus of an individual adsorbent placed at positions along the $z$ axis, centered through the $400 \mu \mathrm{m} \times 400 \mu \mathrm{m}$ face of the matrix structure. Black lines illustrate the surface of the matrix and dashed lines correspond to positions where the adsorbent is completely encapsulated. (d) $\mathrm{CO}_{2}$ adsorption at $308 \mathrm{~K}$ of $\mathrm{NH}_{2}-\mathrm{MIL}-53(\mathrm{Al})$ (adsorbent) and an MMM of $\mathrm{NH}_{2}-\mathrm{MIL}-53(\mathrm{Al})$ within polysulfone (asdorbent@MMM) at $25 \%$ volume fraction, reported by Gascon and coworkers. 21 (d, inset) Predicted average bulk moduli $\left(K_{\mathrm{R}}\right)$ corresponding to $\mathrm{NH}_{2}-\mathrm{MIL}-53(\mathrm{Al})$ and $\mathrm{NH}_{2}-\mathrm{MIL}-53(\mathrm{Al})$ particles dispersed within polysulfone at $21 \%$ volume fraction. 
We have thus shown the effect that concentration of adsorbents and position within an MMM has on their mechanical properties. This has highlighted the changes that encapsulation within a different material can have on the bulk modulus which has a significant impact on flexible adsorption processes such as breathing. Importantly, this approach can be readily applied to predict the flexibility of experimental systems. For example, Gascon and coworkers described an MMM combining flexible $\mathrm{NH}_{2}-\mathrm{MIL}-53(\mathrm{Al})$ and polysulfone.21 The effective bulk modulus of $\mathrm{NH}_{2}$-MIL-53(Al) within this system, for a volume fraction of $21 \%$, is predicted to have an average bulk modulus (Reuss averaging scheme ${ }^{31}$ ) of $5.85 \mathrm{GPa}$, almost $17 \%$ stiffer than that observed for the bare material. This increased stiffness results in a shift of the breathing phenomena to higher pressures as observed by the experimental study, Figure 4 d.

This numerical FEM simulation approach can also be readily extended to anisotropic composite systems, whose behavior is too complex for analytical resolution alone. To illustrate this feature we investigate here composite materials constructed from MIL-53(Al), ${ }^{32}$ a soft porous material which displays NLC: ${ }^{[33}$ when external isotropic pressure is applied, while the overall volume of the system will decrease, some of its dimensions will increase. Notably, NLC has potential applications in pressure sensors, artificial muscles and actuators. 34

Previously, DFT investigations have elucidated the NLC response of MIL-53(Al $)^{35}$ and these results have been used here to simulate the macroscopic consequence of these properties on a particle equivalent to the size of a single crystal. ${ }^{33}$ Simulations employing FEM accurately extend the atomistic NLC phenomenon to single crystal dimensions as demonstrated in Figure 5. Upon application of hydrostatic pressure to a cube structure with the mechanical properties of MIL-53(Al) there is the expected the reduction in volume while, owing to NLC, the width in the $x$-axis increases.

When this NLC particle is encapsulated by even thin layers of a conventional material, matrix 1, the response is attenuated. Figure 6a illustrates the relative width of the $x$-axis of the complete composite for increasing pressure. We find that encapsulation by materials greater than the width of the NLC particle results in complete loss of NLC for the composite. However, for composites constructed with a very thin outer layer (0.1 $\mu \mathrm{m}$ in this case) there remains a small NLC response. In all cases, we find that the particle within the composite retains the NLC behavior as displayed in Figure6 6 . Furthermore, as the ratio surrounding matrix to particle increases so too does the stiffness, evidenced by the drastic reduction in volumetric deformation. This increase in stiffness is attributed to the isotropic encapsulating matrix imparting a greater bulk modulus to the material.

This demonstration of FEM to simulate a composite containing an NLC material highlights the capability of this approach to complex anisotropic materials. Thus unlocking the possibility to investigate combinations of other anisotropic materials providing insight into their macroscopic application. 


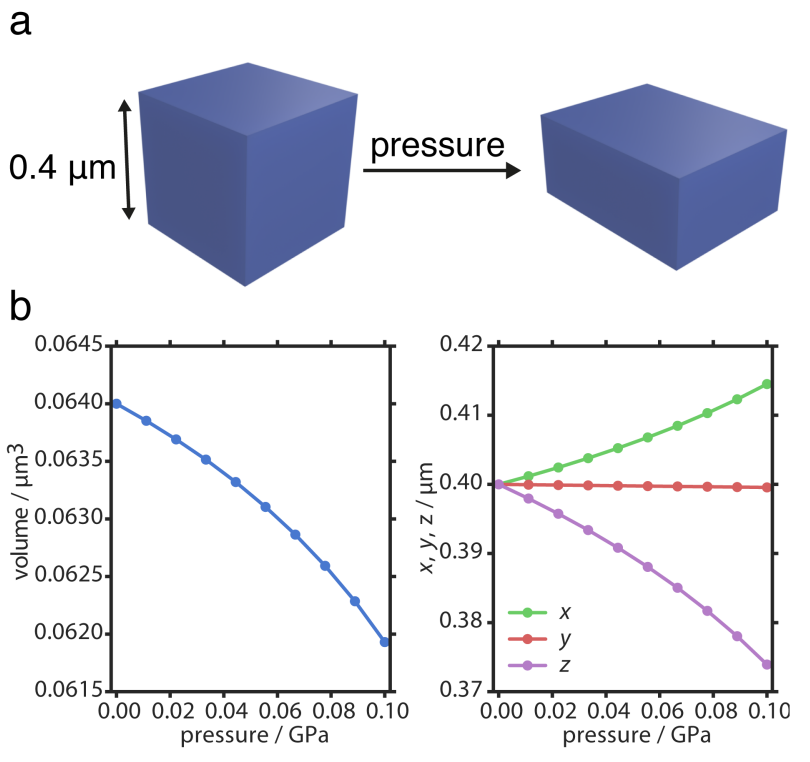

Figure 5: (a) Dimensions of the cube particle used to study the macroscopic NLC response by MIL-53(Al). (b) Changes in the volume and individual dimensions of the particle upon application of increasing hydrostatic pressure.
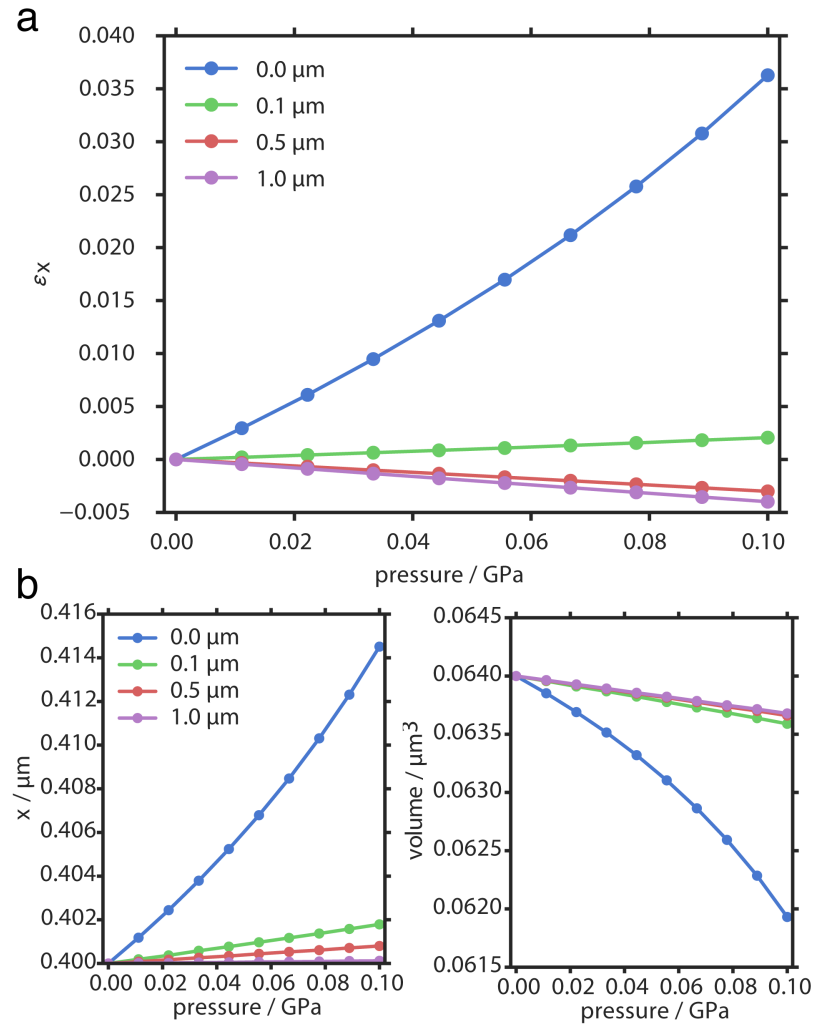

Figure 6: (a) Relative width of the $x$-axis of the total composite material of MIL-53(Al) and matrix 1, for increasing width of the encapsulating material corresponding to the color code. (b) Width of $x$-axis and volume of the NLC material embedded within the composites. 
Our investigation has resulted in insight into the macroscopic mechanical properties of composite microporous materials, an important consideration for the large-scale application of emerging porous phenomena. FEM was used here to simulate increases in bulk modulus of an adsorbent surrounded by another material. The thickness and properties of this encapsulating layer have shown to effect the bulk modulus of the embedded adsorbent and thus has potential to tune the response of flexible adsorption processes. Furthermore, we have demonstrated the application of this methodology to the complex examples of MMMs and NLC materials. These cursory investigations have highlighted the effect position and concentration has on the bulk modulus of the dispersed adsorbent in MMMs and the conditions required to retain an NLC response in a composite. This first application of FEM to the mechanical characterization of soft microporous composites demonstrates its usefulness and opens the door to future investigations to understand specific real-world examples and also towards the development of novel combinations of mechanically anomalous porous materials.

\section{Computational Methods}

COMSOL Multiphysics $5.0^{36}$ was used to create and simulate the $3 \mathrm{D}$ composite models. These simulations employed the MUMPS solver and the default, "normal" settings for mesh size to result in approximately $10^{5}$ tetrahedra in each system. The mechanical properties of the materials modeled are summarized in Table 1. To simulate the stress experienced during guest adsorption, an initial stress was applied to the adsorbent particle(s) and the resulting volumetric strain was measured. The effective bulk modulus was obtained by observing this compression within the linear regime, corresponding in our case to strains smaller than $1 \%$.

Models of MMMs were constructed by randomly dispersing adsorbent spheres within a larger membrane structure. The approach used a MATLAB ${ }^{37}$ script to randomly disperse spheres at a given concentration and subsequently build the geometry in COMSOL, similar to a methodology used by Singh and coworkers. 25 Finally, in the last section, anisotropy was investigated for a cube particle of size $0.4 \mu \mathrm{m}$ with properties corresponding to the stiffness matrix of MIL-53(Al), Table 2. Composites were constructed from this particle surrounded by a cube with properties of matrix 1 of increasing width. The geometry of this cube and corresponding composites were simulated for applied hydrostatic pressure. Examples of input files can be found in the supporting information and the group's data repository at https://github.com/fxcoudert/ citable-data 
Table 2: Stiffness matrix (or matrix of second-order elastic constants) of the anisotropic material MIL-53(Al), ${ }^{32}$ with coefficients given in units of GPa. The values of the elastic constants were obtained using the B3LYP exhange-correlation function with empirical correction for dispersive interactions. $\frac{35}{35}$

$$
\left(\begin{array}{cccccc}
90.85 & 20.41 & 54.28 & 0 & 0 & 0 \\
20.41 & 65.56 & 12.36 & 0 & 0 & 0 \\
54.28 & 12.36 & 33.33 & 0 & 0 & 0 \\
0 & 0 & 0 & 7.24 & 0 & 0 \\
0 & 0 & 0 & 0 & 39.52 & 0 \\
0 & 0 & 0 & 0 & 0 & 8.27
\end{array}\right)
$$

\section{Acknowledgement}

We thank Lydéric Bocquet and Sophie Marbach for fruitful discussions on mechanics of composites and finite element methods. We acknowledge PSL Research University for funding (project DEFORM, grant ANR-10-IDEX-0001-02) and HPC platforms provided by a GENCI grant (x2016087069).

\section{Supporting Information Available}

Examples of the COMSOL input files used for the simulation of the spherical composites, MMMs and anisotropic materials in addition to the MATLAB script used to randomly disperse spheres. This material is available free of charge via the Internet at http://pubs.acs.org/

\section{References}

(1) Horike, S.; Shimomura, S.; Kitagawa, S. Soft Porous Crystals. Nature Chem. 2009, 1, 695-704.

(2) Thallapally, P. K.; Tian, J.; Kishan, M. R.; Fernandez, C. A.; Dalgarno, S. J.; McGrail, P. B.; Warren, J. E.; Atwood, J. L. Flexible (Breathing) Interpenetrated Metal-Organic Frameworks for $\mathrm{CO}_{2}$ Separation Applications. J. Am. Chem. Soc. 2008, 130, 16842-16843.

(3) Horcajada, P.; Serre, C.; Maurin, G.; Ramsahye, N. A.; Balas, F.; Vallet-Regí, M.; Sebban, M.; Taulelle, F.; Férey, G. Flexible Porous Metal-Organic Frameworks for a Controlled Drug Delivery. J. Am. Chem. Soc. 2008, 130, 6774-6780.

(4) Férey, G.; Serre, C. Large Breathing Effects in Three-dimensional Porous Hybrid Matter: Facts, Analyses, Rules and Consequences. Chem. Soc. Rev. 2009, 38, 1380.

(5) Coudert, F.-X. Responsive Metal-Organic Frameworks and Framework Materials: Under Pressure, Taking the Heat, in the Spotlight, with Friends. Chem. Mater. 2015, 27, 1905-1916. 
(6) Neimark, A. V.; Coudert, F.-X.; Boutin, A.; Fuchs, A. H. Stress-Based Model for the Breathing of Metal-Organic Frameworks. J. Phys. Chem. Lett. 2010, 1, 445-449.

(7) Cheng, Y.; Kajiro, H.; Noguchi, H.; Kondo, A.; Ohba, T.; Hattori, Y.; Kaneko, K.; Kanoh, H. Tuning of Gate Opening of an Elastic Layered Structure MOF in $\mathrm{CO}_{2}$ Sorption with a Trace of Alcohol Molecules. Langmuir 2011, 27, 6905-6909.

(8) Serre, C.; Bourrelly, S.; Vimont, A.; Ramsahye, N.; Maurin, G.; Llewellyn, P.; Daturi, M.; Filinchuk, Y.; Leynaud, O.; Barnes, P. et al. An Explanation for the Very Large Breathing Effect of a Metal-Organic Framework during $\mathrm{CO}_{2}$ Adsorption. Adv. Mater. 2007, 19, 2246-2251.

(9) Krause, S.; Bon, V.; Senkovska, I.; Stoeck, U.; Wallacher, D.; Többens, D. M.; Zander, S.; Pillai, R. S.; Maurin, G.; Coudert, F.-X. et al. A Pressure-Amplifying Framework Material with Negative Gas Adsorption Transitions. Nature 2016, 532, 348-352.

(10) Evans, J. D.; Fraux, G.; Gaillac, R.; Kohen, D.; Trousselet, F.; Vanson, J.-M.; Coudert, F.-X. Computational Chemistry Methods for Nanoporous Materials. Chem. Mater. 2017, 29, 199-212.

(11) Qiao, Y.; Wang, K.; Yuan, H.; Yang, K.; Zou, B. Negative Linear Compressibility in Organic Mineral Ammonium Oxalate Monohydrate with Hydrogen Bonding Wine-Rack Motifs. J. Phys. Chem. Lett. 2015, 6, 2755-2760.

(12) Siddorn, M.; Coudert, F.-X.; Evans, K. E.; Marmier, A. A Systematic Typology for Negative Poisson's Ratio Materials and the Prediction of Complete Auxeticity in Pure Silica Zeolite JST. Phys. Chem. Chem. Phys. 2015, 17, 17927-17933.

(13) Evans, J. D.; Coudert, F.-X. Microscopic Mechanism of Chiral Induction in a Metal-Organic Framework. J. Am. Chem. Soc. 2016, 138, 6131-6134.

(14) Zhu, Q.-L.; Xu, Q. Metal-Organic Framework Composites. Chem. Soc. Rev. 2014, 43, 5468-5512.

(15) Bae, T.-H.; Lee, J. S.; Qiu, W.; Koros, W. J.; Jones, C. W.; Nair, S. A High-Performance Gas-Separation Membrane Containing Submicrometer-Sized Metal-Organic Framework Crystals. Angew. Chem. Int. Ed. 2010, 49, 9863-9866.

(16) Bradshaw, D.; Garai, A.; Huo, J. Metal-Organic Framework Growth at Functional Interfaces: Thin Films and Composites for Diverse Applications. Chem. Soc. Rev. 2012, 41, 2344-2381.

(17) Bétard, A.; Fischer, R. A. Metal-Organic Framework Thin Films: From Fundamentals to Applications. Chem. Rev. 2012, 112, 1055-1083. 
(18) Zienkiewicz, O. C.; Taylor, R. L. The Finite Element Method: Solid Mechanics; Butterworth-heinemann, 2000; Vol. 2.

(19) Perez, E. V.; Kalaw, G. J.; Ferraris, J. P.; Balkus, K. J.; Musselman, I. H. Amine-functionalized (Al) MIL-53/ VTEC ${ }^{\mathrm{TM}}$ Mixed-Matrix Membranes for H2/CO2 Mixture Separations at High Pressure and High Temperature. J. Membr. Sci. 2017,

(20) Hsieh, J. O.; Balkus, K. J.; Ferraris, J. P.; Musselman, I. H. MIL-53 Frameworks in Mixed-Matrix Membranes. Micropor. Mesopor. Mat. 2014, 196, 165-174.

(21) Zornoza, B.; Martinez-Joaristi, A.; Serra-Crespo, P.; Tellez, C.; Coronas, J.; Gascon, J.; Kapteijn, F. Functionalized Flexible MOFs as Fillers in Mixed Matrix Membranes for Highly Selective Separation of $\mathrm{CO}_{2}$ from $\mathrm{CH}_{4}$ at Elevated Pressures. Chem. Commun. 2011, 47, 9522.

(22) Coudert, F.-X.; Fuchs, A. H.; Neimark, A. V. Adsorption Deformation of Microporous Composites. Dalton Trans. 2016, 45, 4136-4140.

(23) Mason, J. A.; Oktawiec, J.; Taylor, M. K.; Hudson, M. R.; Rodriguez, J.; Bachman, J. E.; Gonzalez, M. I.; Cervellino, A.; Guagliardi, A.; Brown, C. M. et al. Methane Storage in Flexible Metal-Organic Frameworks with Intrinsic Thermal Management. Nature 2015, 527, 357-361.

(24) Keskin, S.; Sholl, D. S. Selecting Metal Organic Frameworks as Enabling Materials in Mixed Matrix Membranes for High Efficiency Natural Gas Purification. Energy Environ. Sci. 2010, 3, 343.

(25) Singh, T.; Kang, D.-Y.; Nair, S. Rigorous Calculations of Permeation in Mixed-Matrix Membranes: Evaluation of Interfacial Equilibrium Effects and Permeability-based Models. J. Membr. Sci. 2013, 448, 160-169.

(26) Sorribas, S.; Zornoza, B.; Téllez, C.; Coronas, J. Ordered Mesoporous Silica-(ZIF-8) Core-Shell Spheres. Chemical Communications 2012, 48, 9388.

(27) Falcaro, P.; Ricco, R.; Doherty, C. M.; Liang, K.; Hill, A. J.; Styles, M. J. MOF Positioning Technology and Device Fabrication. Chem. Soc. Rev. 2014, 43, 5513-5560.

(28) Park, K. S.; Ni, Z.; Cote, A. P.; Choi, J. Y.; Huang, R.; Uribe-Romo, F. J.; Chae, H. K.; O’Keeffe, M.; Yaghi, O. M. Exceptional Chemical and Thermal Stability of Zeolitic Imidazolate Frameworks. Proceedings of the National Academy of Sciences 2006, 103, 10186-10191.

(29) O'Neill, L. D.; Zhang, H.; Bradshaw, D. Macro-/microporous MOF Composite Beads. Journal of Materials Chemistry 2010, 20, 5720 . 
(30) Sholl, D. S.; Lively, R. P. Seven Chemical Separations to Change the World. Nature 2016, 532, 435-437.

(31) Chung, D. H.; Buessem, W. R. The Elastic Anisotropy of Crystals. Journal of Applied Physics 1967, 38, 2010-2012.

(32) Loiseau, T.; Serre, C.; Huguenard, C.; Fink, G.; Taulelle, F.; Henry, M.; Bataille, T.; Férey, G. A Rationale for the Large Breathing of the Porous Aluminum Terephthalate (MIL-53) Upon Hydration. Chem. Eur. J. 2004, 10, 1373-1382.

(33) Serra-Crespo, P.; Dikhtiarenko, A.; Stavitski, E.; Juan-Alcañiz, J.; Kapteijn, F.; Coudert, F.-X.; Gascon, J. Experimental Evidence of Negative Linear Compressibility in the MIL-53 Metal-Organic Framework Family. CrystEngComm 2015, 17, 276-280.

(34) Cairns, A. B.; Goodwin, A. L. Negative Linear Compressibility. Phys. Chem. Chem. Phys. 2015, 17, 20449-20465.

(35) Ortiz, A. U.; Boutin, A.; Fuchs, A. H.; Coudert, F.-X. Anisotropic Elastic Properties of Flexible MetalOrganic Frameworks: How Soft are Soft Porous Crystals? Phys. Rev. Lett. 2012, 109.

(36) COMSOL, COMSOL Multiphysics 5.0; COMSOL, 2014.

(37) MATLAB, version 8.1.0.604 (R2013a); The MathWorks Inc., 2010. 